\title{
Declining tuberculosis prevalence in Saharia, a particularly vulnerable tribal community in Central India: evidences for action
}

\author{
V. G. Rao ${ }^{1 *}$, J. Bhat ${ }^{1}$, R. Yadav ${ }^{1}$, R. K. Sharma ${ }^{1}$ and M. Muniyandi²
}

\begin{abstract}
Background: In spite of an alarmingly high tuberculosis (TB) burden amongst the Saharia tribe of central India, there is hardly any study to investigate the impact of DOTS implementation on the magnitude of tuberculosis disease and the changes over time. This article present the findings of TB prevalence surveys conducted amongst this indigenous population in two different time periods to know the change in the prevalence of TB.

Methods: A cross sectional survey was conducted among Saharia population in Shivpuri district, Madhya Pradesh during February 2013 to May 2013 and resurvey during March 2015 to July 2015. All individuals ( $\geq 15$ years) were examined for chest symptoms suggestive of TB. Sputum samples were collected from all presumptive TB cases and were confirmed by laboratory examination by Ziehl-Neelsen smear microscopy and solid media culture methods. All detected cases were referred to health facility for anti-tuberculosis treatment as per RNTCP guidelines.

Results: There was significant reduction (trend Chi square 19.97; $O R=1.521 ; p=0.000$ ) in the prevalence of TB at the endline (1995 per 100,000) as compared to baseline (3003 per 100,000). The reduction was significant among males as compared to females (OR 1.55; $p=0.000$ ) and in the age group of $25-34$ years (OR 2.0; $p=0.007$ ) and $45-54$ years (OR 4.39; $p=0.003)$. There was significant reduction in the prevalence in both smear (OR 1.29; $p=0.02)$ and culture positive (OR 1.57; $p=0.000)$ TB at the endline survey.

Conclusion: The study findings highlight a reduction in the prevalence of TB among Saharia tribal population. Further studies are needed to identify the factors associated with reduction in prevalence among this population and also further surveys to monitor the prevalence trend over a period.
\end{abstract}

Keywords: Tuberculosis, Prevalence, Tribal, Saharia, PVTG, Madhya Pradesh, India

\section{Background}

The tribal populations in India are one of the marginalised sections who are constrained by structural and cultural barriers in accessing health services. The magnitude of tuberculosis disease among vulnerable population and the changes over time are useful indicators for understanding the extent of $\mathrm{TB}$ transmission and for monitoring the effectiveness of TB control in the area. Information on these aspects is not available in tribal areas as it is difficult to obtain such information at the community level especially in a large country like India with limited resources.

\footnotetext{
* Correspondence: drvgrao@rediffmail.com

'ICMR - National Institute of Research in Tribal Health, (Indian Council of Medical Research), Nagpur Road, P.O. Garha, Jabalpur 482 003, India Full list of author information is available at the end of the article
}

In India, the tribal population is 104 million constituting $8.6 \%$ of the total population and more than $70 \%$ of these reside in central Indian states of the country [1, 2]. Madhya Pradesh is one of the central Indian states accounting for $21.1 \%$ of tribal population with 46 ethnic groups. Of these, three are notified as Particularly Vulnerable Tribal Groups (PVTGs) [3]. The Saharia tribe is one among these PVTGs living in geographically isolated locations, working mainly as agriculture labours with very low socio economic living conditions. A very high prevalence of infection (20.4\%) and TB disease (1518 per 100,000) has been reported among them $[4,5]$. A recently conducted TB disease prevalence survey in Gwalior district show alarmingly high TB prevalence of 3294 per 100,000 in this community [6]. The high prevalence of PTB was found to

(c) The Author(s). 2019 Open Access This article is distributed under the terms of the Creative Commons Attribution 4.0 International License (http://creativecommons.org/licenses/by/4.0/), which permits unrestricted use, distribution, and 
be associated with malnutrition, poor housing conditions, alcoholism, tobacco smoking and history of asthma. HIV was not found to be a risk factor among them $[7,8]$.

Following a review of the National Tuberculosis Programme (NTP) in 1992, the Government of India initiated the Revised National Tuberculosis Control Programme (RNTCP) in 1993 and adopted internationally recommended Directly Observed Treatment Short-course (DOTS) strategy [9]. RNTCP after being successfully implemented for more than two decades had resulted in significant reduction of TB prevalence and it was demonstrated in Tamil Nadu [10]. With this background, we conducted periodic prevalence surveys to investigate the impact of DOTS implementation in this tribal community residing in hard to reach tribal areas of Shivpuri district of the state. This paper present the findings of the baseline and endline TB prevalence surveys conducted amongst them by the ICMR - National Institute for Research in Tribal Health (NIRTH), Jabalpur.

\section{Methods}

\section{Study area and population}

Saharia is the main tribal community in Shivpuri district, northwest Madhya Pradesh in central India. They generally inhabit small clusters of houses - a hamlet called as 'Saharana' outside the main village. A cross sectional sample survey was conducted among Saharia population in tribal dominated Pohri Block of the district during February 2013 to May 2013 and resurvey during March 2015 to July 2015 using the same methodology. The Directly Observed Treatment, Short-course (DOTS) has been implemented under the Revised National Tuberculosis Control Programme (RNTCP) in the study area since 2004. In addition to tuberculosis case detection under RNTCP at health facilities, a community survey for active tuberculosis detection was undertaken in the study area as epidemiological investigation.

\section{Sample size calculation}

Assuming $25 \%$ precision with 95\% confidence level, design effect of two to adjust for clustering effect and $90 \%$ sample coverage, the sample size of 9225 was calculated based on the reported prevalence of $1518 / 100,000$ bacteriologically positive TB cases among tribal population [5]. Considering the average size of a Saharia hamlet as 300 , it was estimated that about 45 villages would be required to be visited to cover the estimated sample of 9225 adult population (15 years and above).

\section{Sampling design and procedure}

Villages were covered as the primary sampling units. Of the total 254 villages in Pohri Block, 128 were Saharia dominated ( $>70 \%$ Saharia population) villages and 45 villages were randomly selected from these villages.
Additional eight adjoining Saharia villages were also selected during the baseline survey to cover the sample of 9225 individuals. All these 53 villages were covered in the baseline and endline surveys.

\section{Data collection}

Systematic communications with the local tribal leaders were established by the study team to inform them about the purpose of our survey. Study team also conducted community meetings prior to the survey. After this sensitization, house to house census was carried out by our trained field investigators and household information was recorded in an individual card in a precoded form. A baseline survey was conducted among all individuals aged 15 years and above to screen for symptoms suggestive of TB viz. cough for $\geq 2$ weeks, fever $\geq 1$ month, chest pain for $\geq 1$ month and history of haemoptysis. Individuals remaining absent for symptom inquiry were revisited the same day or on subsequent days until at least $90 \%$ coverage was achieved. Persons having any of these symptoms were considered as chest symptomatic and two sputum specimens were collected for smear and culture examination. One sputum sample was collected on the spot and the overnight sample was collected in the morning in sterilized McCartney's bottles from chest symptomatics as well as from asymptomatic individuals having a prior history of receiving anti $\mathrm{TB}$ treatment. The samples were sent to the ICMR-NIRTH laboratory, Jabalpur for smear and culture. ZN method was used to prepare smears from the concentrated samples. For culture examination, the sputum samples were kept in refrigerated condition till bacteriological investigations were carried out. The samples were processed and inoculated on 2 slopes of Lowensten Jensen (LJ) medium. The cultures were incubated and examined every week for the presence of mycobacterial colonies for a period of 8 weeks. In case of no growth or contamination, the cultures were discarded. M. Tuberculosis growth was confirmed using niacin test, $68^{\circ} \mathrm{C}$ catalase test and growth on LJ with PNB $(500 \mu \mathrm{g} / \mathrm{ml})$. Individuals having positive smear and/or culture were considered to have tuberculosis and were referred to the health facility for anti-tuberculosis treatment as per RNTCP guidelines.

\section{Data management}

All the completed cards and laboratory reports were scrutinized, checked for completeness and any incomplete forms sent back to the field for completeness. All the data were computerized in entry package developed on Census and Survey Processing System (CSPro) and SPSS was used for analysis. A pulmonary TB case was defined as an individual whose sputum was positive for AFB by ZN microscopy and/or growth of M. tuberculosis by culture examination. Prevalence of TB was estimated 
per 100,000 population and univariate analysis age, sex wise stratification was done. Statistical tool used was univariate analysis. Chi square test of significance was used to test differences in distribution of study population. Trend Chi square was used to test the prevalence differences in different surveys. The $p$ value of $<0.05$ was considered as the level of statistical significance.

\section{Ethical issues}

The study was approved by the Institutional Ethics Committee at ICMR - National Institute of Research in Tribal Health, Jabalpur. The trained field investigators approached eligible individuals and explained the procedures, risks and benefits of the study in the local language. Written informed consent was obtained from all individuals willing to participate. All the patients diagnosed during the survey were referred to RNTCP and free counselling was provided to complete full course of the treatment.

\section{Results}

\section{Coverage and TB cases}

Of the total 10,300 and 10,573 individuals eligible for screening for baseline and endline surveys 9756 (94.7\%) and 9775 (92.5\%) individuals belonging to Saharia tribal community were screened (Table 1). Of the individuals screened for symptoms, $1463(15.0 \%)$ and 945 (9.7\%) were found to be symptomatic and sputum was collected from 1430 (97.7\%) and 908 (96.1\%) in baseline and end line surveys respectively. The coverage of $>90 \%$ was achieved during both the surveys. At the baseline and endline surveys, a total of 9756 and 9775 population was screened and 293 and $195 \mathrm{~TB}$ cases were detected respectively (Fig. 1), i.e. 50 Saharia individuals needed to be screened for detecting one PTB case in the endline survey as compared to 33 individuals in the baseline survey. The number of cases detected among male population was three times higher as compared to cases detected among females in both the surveys (231\& 62 in baseline, 149\& 46 in endline).

\section{Prevalence of pulmonary tuberculosis}

The overall prevalence among tribal population was found to be 3003 (95\% Confidence Interval (CI): 2296-3710) per 100,000 in the baseline and 1995 (95\% CI, 1323-2667) per 100,000 in the endline survey. There was significant reduction (trend Chi square 19.97; $\mathrm{OR}=1.521 ; p=0.000)$ in the prevalence of $\mathrm{TB}$ at the endline as compared to baseline (Fig. 2). In both the surveys, higher prevalence was observed among males compared to females (baseline 4832 vs 1246/ 100,000 population; endline 3170 vs $906 / 100,000$ population). The reduction in prevalence was $1662((34.4 \%)$ among males and 340 (27.3\%) among females (Fig. 3). There was significant prevalence reduction among males as compared to females (OR 1.55; $p=0.000$ ). A significantly higher prevalence was consistently observed among males compared to females for smear, culture and bacteriologically positive PTB.

The prevalence of smear positive PTB was estimated to be 1978 and 1514 per 100,000 during baseline and endline respectively. The prevalence of culture positive PTB was estimated to be 2378 and 1494 per 100,000 during baseline and endline respectively. There was significant reduction in the prevalence in both smear (OR 1.29; $p=0.02$ ) and culture positive (OR 1.57; $p=0.000$ ) $\mathrm{TB}$ at the endline as compared to baseline survey. The significant reduction in the prevalence in both smear and culture positive TB was also seen among males as compared to females (change in smear prevalence 25\% vs $12.6 \%$; culture positive $37.9 \%$ vs $31.2 \%$ respectively). The prevalence reduction was higher (more than $45 \%$ )

Table 1 Coverage of study population at baseline and endline surveys

\begin{tabular}{|c|c|c|c|c|c|c|}
\hline & \multicolumn{3}{|l|}{ Baseline survey 2012-13 } & \multicolumn{3}{|l|}{ Endline survey 2014-15 } \\
\hline & No. eligible to examine & No. examined & Coverage (\%) & No. eligible to examine & No. examined & Coverage (\%) \\
\hline \multicolumn{7}{|c|}{ Sex } \\
\hline Male & 5157 & 4781 & 92.7 & 5384 & 4700 & 87.3 \\
\hline Female & 5143 & 4975 & 96.7 & 5189 & 5075 & 97.8 \\
\hline \multicolumn{7}{|c|}{ Age (years) } \\
\hline $15-24$ & 2951 & 2853 & 96.7 & 2624 & 2531 & 96.5 \\
\hline $25-34$ & 2742 & 2616 & 95.4 & 3208 & 3015 & 94.0 \\
\hline $35-44$ & 1920 & 1783 & 92.9 & 1989 & 1837 & 92.4 \\
\hline $45-54$ & 1499 & 1391 & 92.8 & 1524 & 1367 & 89.7 \\
\hline$\geq 55$ & 1188 & 1113 & 93.7 & 1228 & 1025 & 83.5 \\
\hline Total & 10,300 & 9756 & 94.7 & 10,573 & 9775 & 92.5 \\
\hline
\end{tabular}


Baseline survey

(February 2013 to May 2013)

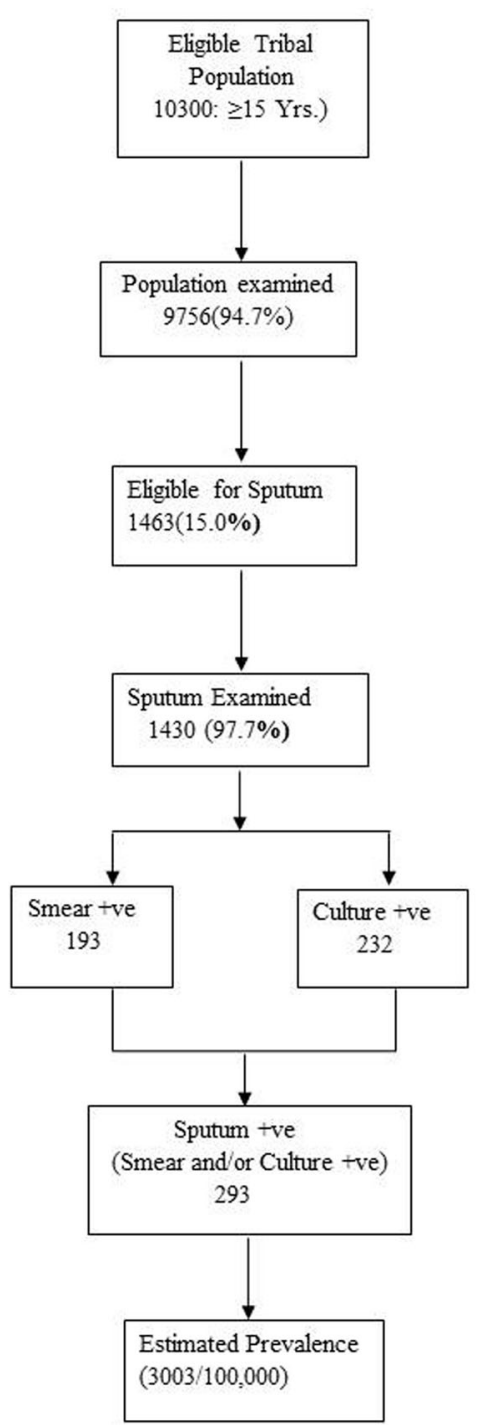

Endline survey

(March 2015 to July 2015)

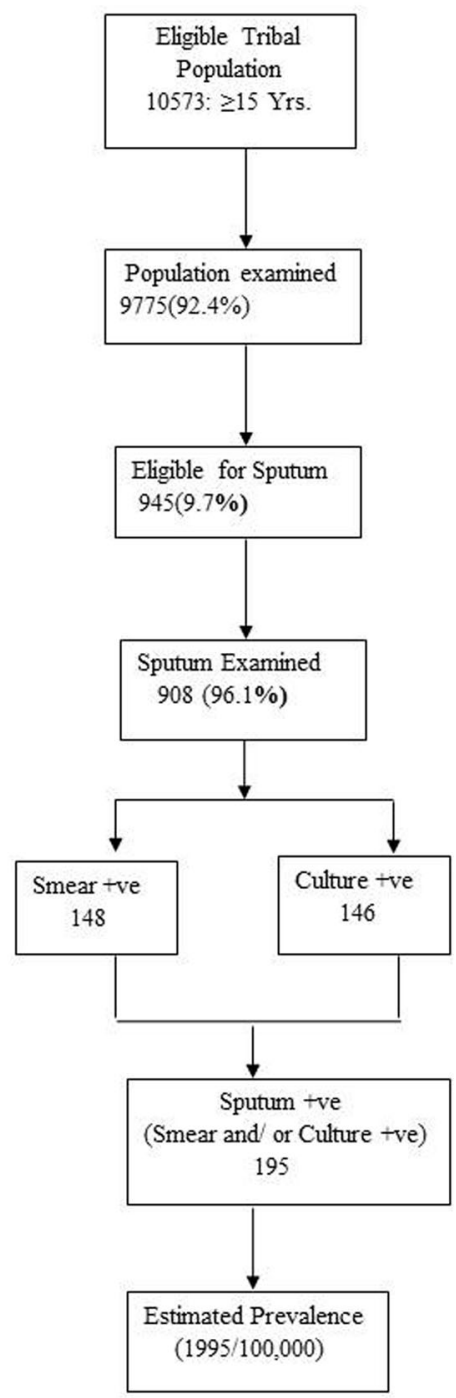

Fig. 1 Process of baseline and endline prevalence surveys conducted among Saharia population, Shivpuri district, Madhya Pradesh

among adult population aged $25-54$ as compared to younger population. However, an increase in culture positive TB in the endline (2965 to 3024/100000) among population aged 55 years and above (Table 2). The prevalence of PTB increased with the age in both the baseline and endline surveys (Fig. 4). The reduction of TB was significant in the age group of 25-34 years (OR $2.0 ; p=0.007$ ) and $45-54$ years (OR 4.39; $p=0.003$ ).

\section{Discussion}

The salient finding from this study is the decreasing trend in prevalence of PTB in Saharia tribal community of central Indian state of Madhya Pradesh. Analysis of data on burden of disease caused by TB and the effectiveness of programmatic efforts to reduce this burden are crucial for public health action [11]. Further, this analysis is essential for programme evaluation which helps guide decisions about TB control management activities and policy. It supports TB control programme managers to monitor trends in the number and distribution of $\mathrm{TB}$ cases across the region. This enables TB control activities to report on the country's TB epidemic and progress in reaching $\mathrm{TB}$ control goals and objectives. It also helps TB control programme managers to develop targeted national strategies and budget plans.

The current finding from community based prevalence survey highlight alarmingly high TB disease burden among Saharia tribe as compared to the national average 


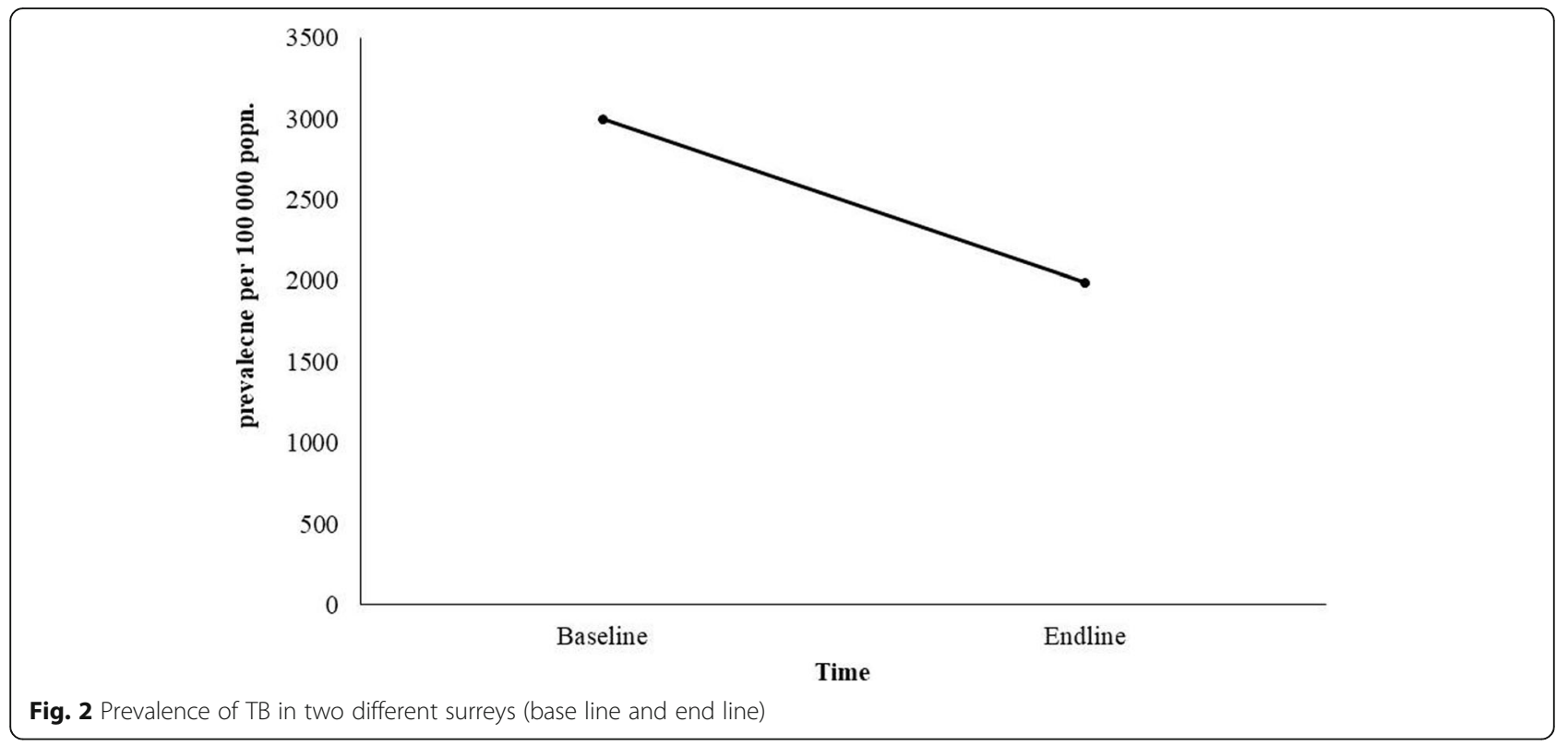

and different population groups within the country [5]. This finding corroborate with the global evidences of high burden of TB among indigenous population [12]. The incidence of TB among indigenous peoples in Latin America was 1000/100000 and it was around 20 times higher than the incidence in the general population [13]. Among Yanomami people found the incidence of TB to be $2133 / 100000$, or 37 times higher than in the surrounding non-indigenous population [14]. Indigenous people living in Cotopaxi Province in Ecuador's highlands had TB prevalence rates reaching 6700/100000 [15].
It is encouraging that the repeat survey showed reduction in TB prevalence suggesting that efforts to improve TB control through active case finding was one of the important contributory factor in reducing the prevalence. The study from south India reported substantial reduction in TB prevalence in 5 years with DOTS implementation along with active case finding in the population $[16,17]$. The tribal population in India also has access to free DOTS under RNTCP. This could be a plausible factor behind decreasing trend of TB among this vulnerable population. The analysis of national

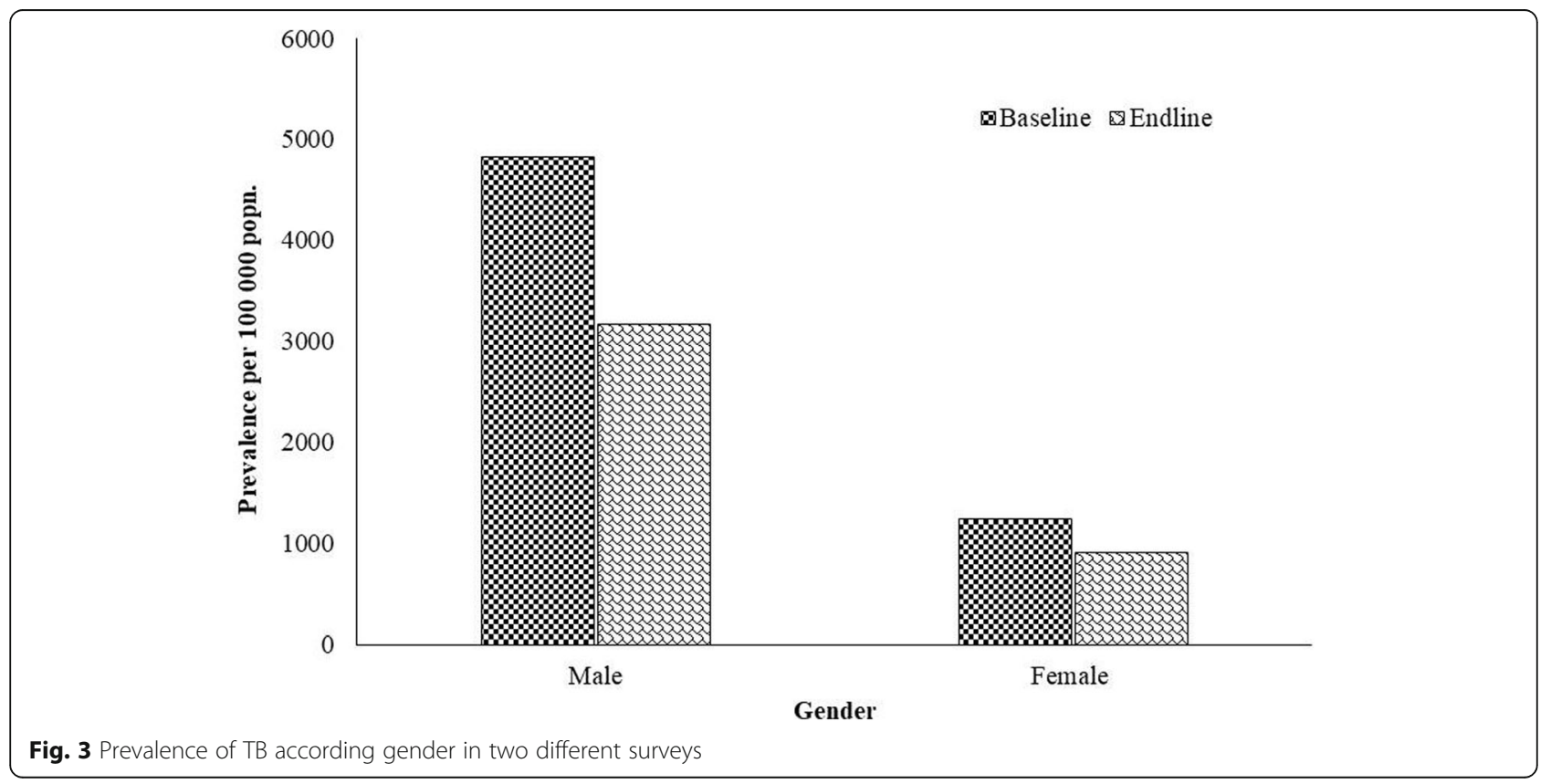


Table 2 Prevalence of smear positive and culture positive TB at two different time points

\begin{tabular}{|c|c|c|c|c|c|c|c|c|}
\hline & \multicolumn{2}{|c|}{ Number examined } & \multicolumn{2}{|c|}{ Number of Pulmonary TB } & \multicolumn{2}{|c|}{ Prevalence Per 100,000 popn. } & \multicolumn{2}{|c|}{ Change in prevalence } \\
\hline & Baseline & Endline & Baseline & Endline & Baseline & Endline & No & $\%$ \\
\hline \multicolumn{9}{|c|}{ Smear-positive } \\
\hline \multicolumn{9}{|l|}{ Sex } \\
\hline Male & 4781 & 4700 & 156 & 115 & 3263 & 2447 & 816 & 25.0 \\
\hline Female & 4975 & 5075 & 37 & 33 & 744 & 650 & 93 & 12.6 \\
\hline \multicolumn{9}{|c|}{ Age (years) } \\
\hline $15-24$ & 2853 & 2531 & 24 & 17 & 841 & 672 & 170 & 20.2 \\
\hline $25-34$ & 2616 & 3015 & 42 & 37 & 1606 & 1227 & 378 & 23.6 \\
\hline $35-44$ & 1783 & 1837 & 45 & 39 & 2524 & 2123 & 401 & 15.9 \\
\hline $45-54$ & 1391 & 1367 & 51 & 31 & 3666 & 2268 & 1399 & 38.1 \\
\hline$\geq 55$ & 1113 & 1025 & 31 & 24 & 2785 & 2341 & 444 & 15.9 \\
\hline \multicolumn{9}{|c|}{ Culture positive } \\
\hline \multicolumn{9}{|l|}{ Sex } \\
\hline Male & 4781 & 4700 & 185 & 113 & 3869 & 2404 & 1465 & 37.9 \\
\hline Female & 4975 & 5075 & 47 & 33 & 945 & 650 & 294 & 31.2 \\
\hline \multicolumn{9}{|c|}{ Age (years) } \\
\hline $15-24$ & 2853 & 2531 & 24 & 17 & 841 & 672 & 170 & 20.2 \\
\hline $25-34$ & 2616 & 3015 & 52 & 31 & 1988 & 1028 & 960 & 48.3 \\
\hline $35-44$ & 1783 & 1837 & 62 & 34 & 3477 & 1851 & 1626 & 46.8 \\
\hline $45-54$ & 1391 & 1367 & 61 & 33 & 4385 & 2414 & 1971 & 45.0 \\
\hline$\geq 55$ & 1113 & 1025 & 33 & 31 & 2965 & 3024 & -59 & -2.0 \\
\hline
\end{tabular}

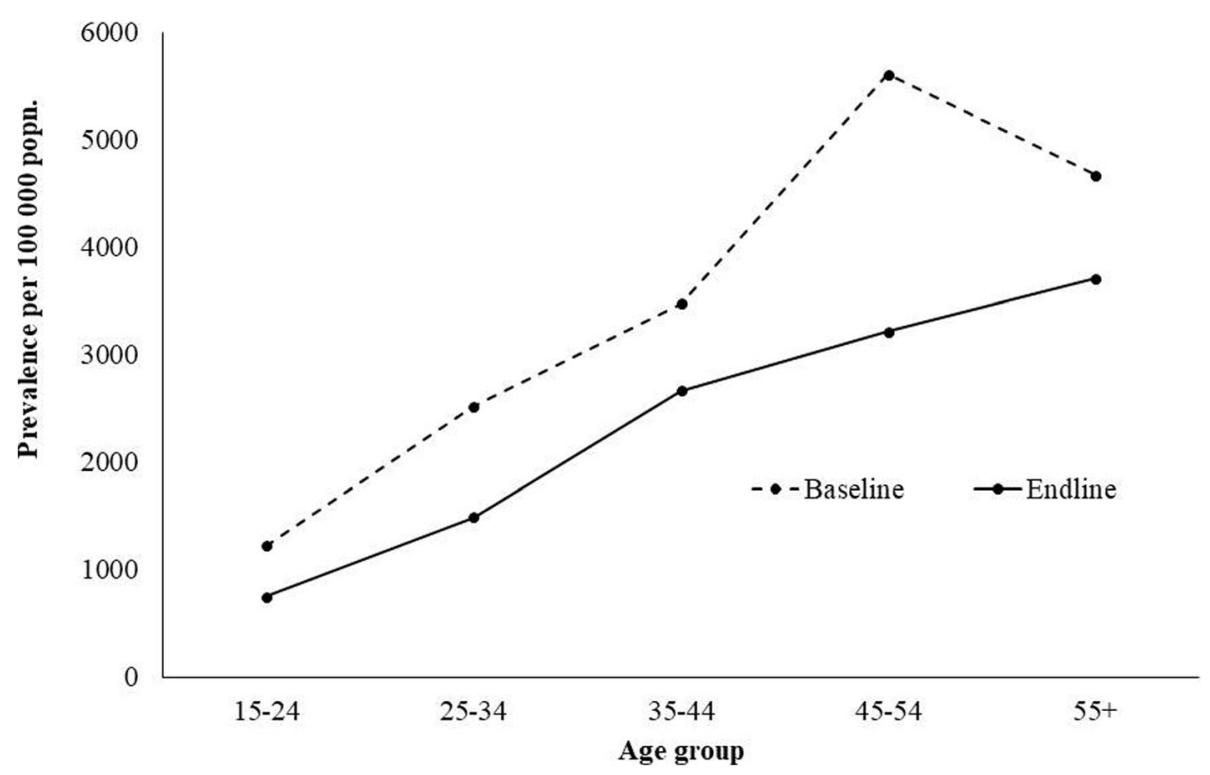

Fig. 4 Prevalence of TB in two different surreys in different age group 
surveys conducted during 1990-2012 in Asia show 50\% decrease in TB disease within a period of 10 years with some countries showing even greater decline with the available intervention techniques and methods [18].

However, the reduction among elderly population is less as compared to other age group particularly culture positive TB. It has been reported that smear positive TB is less in elderly and there are more chances for adverse drug reactions, default or death of newly diagnosed TB cases in this age group $[19,20]$. Policy makers, in consultation with all the stakeholders, should consider developing specific intervention strategies for TB management in this population group. At the same time, the studies are also required to understand the effect of such interventions on the possible concentration of cases in this group. Given that TB prevalence was high among the elderly, there is a need to step up efforts to manage this vulnerable population.

The current study finding of reduction in TB prevalence occurred in a very short period, whereas the findings from four countries (Cambodia, China, the Philippines and Republic of Korea) demonstrated that approximately $50 \%$ reductions in TB prevalence can be achieved within 10 years [21-23]. Our findings could be understood in different programmatic context. India's RNTCP's DOTS strategy covered the entire country in 2006 and considering the high burden of TB among tribal population and structural barrier for diagnosis and treatment of TB in tribal areas, a tribal action plan was developed and implemented in parallel under RNTCP since 2005. Under the tribal action plan, strengthening and expansion of additional health facilities and involvement of community health workers was ensured. In addition, among Saharia population in Shivpuri district, IEC campaigns and door-to-door active TB case detection survey were implemented. The reduction in TB burden among this population could be due to the cumulative effect of all these interventions.

The study population is economically poor, socially marginalized, living far away from the main locality. There are numerous challenges that this population face in accessing healthcare facility which includes poverty, shortages of healthcare workers, non-availability of medicines, higher transportation cost, work absenteeism, lack of awareness on health facility and availability of treatment. Under the RNTCP Tribal Plan, additional decentralization of diagnosis and treatment services and special incentives to the health workers for tribal areas are provided [24]. The programme performance in the area has shown gradual improvement since implementation of RNTCP with an increase in the case detection rate from $121 \%$ in 2005 to $159 \%$ in 2017 and in the cure rate from $67 \%$ in 2005 to $83 \%$ in 2017 [25, 26]. Way back in 1989, Styblo had also highlighted the challenge before the policy makers that a significant cure rate among smear positive pulmonary $\mathrm{TB}$ cases is pre-requisite to achieve overall improvement in TB burden in developing countries [23]. It is important to have regular and effective monitoring in place in order to understand the effects of the intervention strategies - the improvements or otherwise in disease trend. The surveys carried out at regular intervals may also help in understanding the trend.

\section{Limitations of the study}

The information on socioeconomic change in the community was not available and so its possible impact could not be assessed. Moreover, this evidence is generated from the Saharia tribe where HIV infection and MDR-TB are not highly prevalent $[8,27]$. Our conclusion therefore, cannot be generalized to all areas, especially to those with a high prevalence of HIV infection or MDR-TB and non-tribes.

\section{Conclusion}

In summary, our study has shown that there is a reduction in prevalence of TB among Saharia tribal population. The factors which led to the reduction in prevalence between the study periods need to be further studied. In general, there is a need for effective public health strategies for intervening disproportionate $\mathrm{TB}$ burden among Saharias, who live in resource poor settings.

\section{Abbreviations \\ AFB: Acid-Fast Bacilli; CSPro: Census and Survey Processing System; DOTS: Directly Observed Treatment Short-course; HIV: Human immunodeficiency virus; ICMR : Indian Council of Medical Research; IEC: Information, education and communication; LJ: Lowensten Jensen; MDR- TB: Multi-Drug-Resistant Tuberculosis; NIRTH: National Institute of Research in Tribal Health; NTP: National Tuberculosis Programme; PTB: Pulmonary tuberculosis; PVTG: Particularly Vulnerable Tribal Group; RNTCP: Revised National Tuberculosis Control Programme; SPSS: Statistical package for the social sciences; TB: Tuberculosis; ZN: Ziehl-Neelsen}

\section{Acknowledgements}

The authors gratefully acknowledge the contributions of the State Tuberculosis Officer, District Tuberculosis Officer, the WHO/RNTCP consultant, Block Medical Officers and field staff in the district. We thank our study subjects for their cooperation throughout the study. The assistance provided by Ms. Preeti Tiwari, Mrs. Priyashri Tiwari Pandey and Ms. Pushpa Vishwakarma for the entry, verification and analysis of the data is acknowledged. Thanks are also due to the laboratory and field staff involved in the study.

\section{Funding}

The study was supported by the Indian Council of Medical Research, New Delhi. The funding agency had no role in the design of the study; collection, analysis and interpretation of data and in writing the manuscript.

\section{Availability of data and materials}

The datasets used and/or analysed during the current study available from the corresponding author on reasonable request.

\section{Authors' contributions}

VGR, JB, RKS designed the study protocol; VGR, RY actively participated in the field work for data collection; JB carried out laboratory examination including quality check. VGR, MM, RKS carried out analysis and interpretation of the data; VGR, JB, RY, MM, RKS drafted the manuscript. All authors read and approved the final manuscript. 


\section{Ethics approval and consent to participate}

The study was approved by the Institutional Ethics Committee at ICMR National Institute for Research in Tribal Health, Jabalpur. Written informed consent was obtained from all individuals willing to participate. The written assent and permission from parents/guardians was obtained for participants under 16 years of age.

\section{Consent for publication}

Written informed consent/assent was obtained from the participants for publication.

\section{Competing interests}

The authors declare that they have no competing interests.

\section{Publisher's Note}

Springer Nature remains neutral with regard to jurisdictional claims in published maps and institutional affiliations.

\section{Author details \\ 'ICMR - National Institute of Research in Tribal Health, (Indian Council of Medical Research), Nagpur Road, P.O. Garha, Jabalpur 482 003, India. ${ }^{2}$ ICMR -National Institute for Research in Tuberculosis, (Indian Council of Medical Research), No. 1, Mayor Sathiyamoorthy Road, Chetpet, Chennai 600031,} India.

Received: 6 August 2018 Accepted: 13 February 2019

Published online: 20 February 2019

\section{References}

1. Government of India. Annual Report 2016-17. Ministry of tribal affairs, Government of India. 2017. https://www.tribal.nic.in/writereaddata/ AnnualReport/AnnualReport2016-17.pdf.

2. Government of India. Statistical profile of scheduled tribes in India. Ministry of tribal affairs. Government of India. 2013. https://tribal.nic.in/ST/ StatisticalProfileofSTs2013.pdf

3. Tewari DN. Primitive tribes of Madhya Pradesh - strategy for development New Delhi: Government of India, Ministry of Home Affairs, Tribal Development Division; 1984.

4. Rao VG, Gopi PG, Yadav R, Sadacharam K, Bhat J, Subramani R, et al. Tuberculosis infection in Saharia, a primitive tribal community of Central India. Trans Trop Med Hyg. 2008;102(9):898-904.

5. Rao VG, Gopi PG, Bhat J, Selvakumar N, Yadav R, Tiwari B, et al. Pulmonary tuberculosis : a public health problem amongst Saharia, a primitive tribe of Madhya Pradesh, central India. Int J Infect Dis. 2010;14(8):e713-6. https://doi. org/10.1016/j.jij.2010.02.2243 Epub 2010 Jun 3.

6. Rao VG, Bhat J, Yadav R, Muniyandi M, Sharma R, Bhondeley MK. Pulmonary tuberculosis - a health problem amongst Saharia tribe in Madhya Pradesh. Indian J Med Res. 2015;141:630-5.

7. Rao VG, Bhat J, Yadav R, Muniyandi M, Sharma R. Bhondeley MK. A comparative study of the risk factors for pulmonary tuberculosis in Saharia tribe of Madhya Pradesh, India. Trans R Soc Trop Med Hyg. 2018;112:272-8.

8. Bhat J, Rao VG, Sharma RK, Muniyandi M, Yadav R, Bhondley MK. Investigation of the risk factors for pulmonary tuberculosis: a case- control study among Saharia tribe in Gwalior district, Madhya Pradesh, India. Indian J Med Res. 2017;146:97-104.

9. WHO Global Tuberculosis Programme. Framework for effective tuberculosis control. WHO/TB/94.179. Geneva: WHO; 1994.

10. Subramani R, Radhakrishna S, Frieden TR, Kolappan C, Gopi PG, Santha $T$, et al. Rapid decline in prevalence of pulmonary tuberculosis after DOTS implementation in a rural area of South India. Int J Tuberc Lung Dis. 2008;12(8):916-20.

11. World Health Organization. Understanding and using tuberculosis data. World Health Organization. Geneva: WHO/HTM/TB/2014.09; 2014.

12. Tollefson D, Bloss E, Fanning A, Redd JT, Barker K, McCray E. Burden of tuberculosis in indigenous peoples globally: a systematic review. Int J Tuberc Lung Dis. 2013;17(9):1139-50.

13. Escobar AL, Coimbra CE Jr, Camacho LA, Portela MC. Tuberculosis among indigenous populations in Rondonia, Amazonia, Brazil. Cad SaudePublica. 2001;17:285-98.
14. Levino A, de Oliveira RM. Tuberculosis among the Indian population in Sao Gabriel da Cachoeira, Amazonas State, Brazil. Cad Saude Publica. 2007;23:1728-32.

15. Romero-Sandoval NC, Flores-Carrera OF, Sánchez-Pérez HJ, Sánchez-Pérez I, Mateo MM. Pulmonary tuberculosis in an indigenous community in the mountains of Ecuador. Int J Tuberc Lung Dis. 2007;11:550-5.

16. Subramani R, Radhakrishna S, Frieden TR, Kolappan C, Gopi PG, Santha $\mathrm{T}$, et al. Rapid decline in prevalence of pulmonary TB after DOTS implementation in a rural area of South India. Int J Tuberc Lung Dis. 2008;12:916-20.

17. Kolappan C, Subramani R, Radhakrishna S, Santha T, Wares F, Baskaran D, et al. Trends in the prevalence of pulmonary tuberculosis over a period of seven and half years in a rural community in South India with DOTS. Indian J Tuberc. 2013;60:168-76.

18. Onozaki I, Law I, Sismanidis C, Zignol M, Glaziou P, Floyd K. National tuberculosis prevalence surveys in Asia, 1990-2012: an overview of results and lessons learned. Tropical Med Int Health. 2015;20(9):1128-45.

19. Pardeshi G, Deshmukh D. Disease characteristics and treatment outcomes in elderly tuberculosis patients on DOTS. Indian J Community Med. 2007;32:292-4.

20. Velayutham BR, Nair D, Chandrasekaran V, Raman B, Sekar G, Watson B, et al. Profile and response to anti-tuberculosis treatment among elderly tuberculosis patients treated under the TB control programme in South India. PLoS One. 2014;9(3):e88045. https://doi.org/10.1371/journal.pone.0088045.

21. Wang L, Zhang H, Ruan Y, Chin DP, Xia Y, Cheng $S$, et al. Tuberculosis prevalence in China, 1990-2010; a longitudinal analysis of national survey data. Lancet. 2014:383:2057-64.

22. Mao TE, Okada K, Yamada N, Peou S, Ota M, Saint S, et al. Cross-sectional studies of tuberculosis prevalence in Cambodia between 2002 and 2011. Bull World Health Organ. 2014;92:573-81.

23. Styblo K. Overview and epidemiologic assessment of the current global tuberculosis situation with an emphasis on control in developing countries. Rev Infect Dis. 1989;11(Suppl 2):S339-46.

24. Central TB Division, Government of India. Tribal action plan proposed for the World Bank assisted RNTCP II Project. New Delhi: Ministry of Health and Family welfare, Government of India; 2005.

25. Central TB Division, Government of India. TB INDIA 2006. RNTCP status report. New Delhi: Directorate General of Health Services, Ministry of Health and Family Welfare; 2006.

26. Central TB Division, Government of India. INDIA TB REPORT 2018. Annual status report. New Delhi: Revised National TB Control Programme, Directorate General of Health Services, Ministry of Health and Family Welfare; 2018.

27. Bhat J, Rao VG, Yadav R, Muniyandi M, Sharma R, Karfarma C, et al. Situation of drug resistant tuberculosis in Saharia tribe of central India. Indian J Med Res. 2015;141:636-9.

Ready to submit your research? Choose BMC and benefit from:

- fast, convenient online submission

- thorough peer review by experienced researchers in your field

- rapid publication on acceptance

- support for research data, including large and complex data types

- gold Open Access which fosters wider collaboration and increased citations

- maximum visibility for your research: over $100 \mathrm{M}$ website views per year

At BMC, research is always in progress.

Learn more biomedcentral.com/submissions 